\title{
Comparative Performance Analysis of SALT and PEPPER Noise Removal
}

\author{
Sakshi Tiwari $^{1}$, Prof. Akhilesh Bansiya ${ }^{2}$, Prof. Raj Kumar Paul ${ }^{3}$ \\ ${ }^{I}$ (Research scholar,M.tech in Computer science, VIT Bhopal /RGPV,India) \\ ${ }^{2}$ (Deppt. Of Computer science VIT Bhopal/RGPV,India) \\ ${ }^{3}$ (HOD, deppt. Of Computer science VIT Bhopal/RGPV,India)
}

\begin{abstract}
Noise is an important factor which when get added to an image reduces its quality and appearance. So in order to enhance the image qualities, it has to be removed with preserving the textural information and structural features of image. There are different types of noises exist who corrupt the images. Selection of the de-noising algorithm is application dependent. Noise removal from a contaminated image signal is a prominent field of research and many researchers have suggested a large number of algorithms and compared their results. The main thrust on all such algorithms is to remove impulsive noise while preserving image details. These schemes differ in their basic methodologies applied to suppress noise. Some schemes utilize detection of impulsive noise followed by filtering whereas others filter all the pixels irrespective of corruption. In this section an attempt has been made for a detail literature review on the reported articles and studies their performances through computer simulation. We have classified the schemes based on the characteristics of the filtering schemes and described are below. At the end of paper, a comparative study of all these algorithms in context of performance evaluation is done and concluded with several promising directions for future research work.
\end{abstract}

Keywords: Noise, Textural information, Image de-noising algorithm.

\section{Introduction}

A very large portion of digital image processing is deployed in image restoration. Image restoration is the removal or reduction of degradations which occurred while the image is being obtained [1]. Image processing is an important area in the information industry. A crucial research is how to filter noise caused by the nature, system and processing of transfers and so on. Image de-noising has been one of the most important and widely studied problems in image processing and computer vision. The need to have a very good image quality is increasingly required with the advent of the new technologies in a various areas such as multimedia, medical image analysis, aerospace, video systems and others. Indeed, the acquired image is often marred by noise which may have a multiple origins such as: thermal fluctuations; quantify effects and properties of communication channels [2].A noise is introduced in the transmission medium due to a noisy channel, errors during the measurement process and during quantization of the data for digital storage. Each element in the imaging chain such as lenses, film, digitizer, etc. contributes to the degradation .Image de-noising is often used in the field of

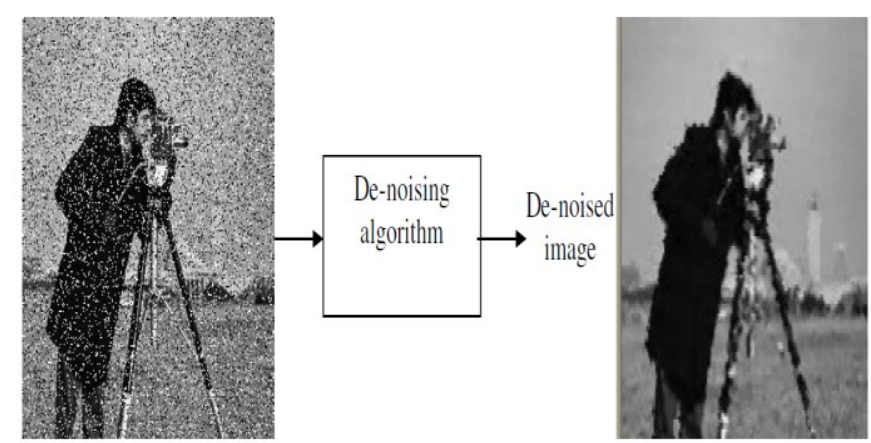

Figure - 1 Image De-noising

Photo-graphy or publishing where an image was somehow degraded but needs to be improved before it can be printed. Image de-noising finds applications in fields such as astronomy where there solution limitations are severe, in medical imaging where the physical requirements for high quality imaging are needed for analyzing images of unique events, and in forensic science where potentially useful photographic evidence is sometimes of extremely bad quality [2].

A two-dimensional digital image can be represented as a 2-dimensional array of data $\mathrm{s}(\mathrm{x}, \mathrm{y})$, where 
$(\mathrm{x}, \mathrm{y})$ represent the pixel location. The pixel value corresponds to the brightness of the image at location $(\mathrm{x}, \mathrm{y})$. Some of the most frequently used image types are binary, gray-scale and color images [3]. Binary images are the simplest type of images and can attain only two discrete values, black and white. Black is represented with the value ' 0 ' while white with ' 1 '. Normally a binary image is generally created from a gray-scale image. A binary image finds applications in computer vision areas where the general shape or outline information of the image is needed. They are also referred to as $1 \mathrm{bit} / \mathrm{pixel}$ images.

The goal of image de-noising is to estimate a clean version of a given noisy image, utilizing prior knowledge on the statistics of natural images. The problem has been studied intensively with considerable progress made in recent years. The challenge in evaluating such limits is that constructing proper models of natural image statistics is a long standing and yet unsolved problem. This raises the question of whether the error rates of current de-noising algorithms can be reduced much further. At the more challenging cases of very large patch sizes or very small noise levels, we only get a lower bound on the best possible de-noising error.

\section{Noise Modal}

Two common types of the impulse noise are the Fixed-Valued Impulse Noise (FVIN), also known as Salt and-Pepper Noise (SPN), and the Random-Valued Impulse Noise (RVIN). They differ in the possible values which noisy pixels can take [6]. The FVIN is commonly modeled by -

$\left(Y_{i j}\right)=\left\{\begin{array}{l}X_{i, j} \text { with probabilty } p \\ (0,255) \text { with probabilty } 1-p\end{array}\right\} \ldots \ldots \ldots \ldots . . . .(1)$

Where $x(i, j)$ and $y(i, j)$ denote the intensity value of the original and corrupted images at coordinate $(i, j)$, respectively and $\mathrm{p}$ is the noise density. This model implies that the pixels are randomly corrupted by two fixed extreme values, 0 and 255 (for 8-bit grey-scale images), with the same probability.

\section{Classification Of De-Noising Algorithms}

On the basis of Fig.-1, it is obvious that there are two basic approaches of image de-noising, spatial filtering methods and transform domain filtering methods.

\section{A. Spatial Filtering}

A traditional way to remove noise from image data is to employ spatial filters. Spatial filters can be further classified into non-linear and linear filters.

\section{Non-Linear Filters}

With non-linear filters, the noise is removed without any attempts to explicitly identify it. Spatial filters employ a low pass filtering on groups of pixels with the assumption that the noise occupies the higher region of frequency spectrum. Generally spatial filters remove noise to a reasonable extent but at the cost of blurring images which in turn makes the edges in pictures invisible. In recent years, a variety of nonlinear median type filters such as weighted median [8], rank conditioned rank selection [9], and relaxed median [10] have been developed to overcome this drawback.

\section{Linear Filters}

A mean filter is the optimal linear filter for Gaussian noise in the sense of mean square error. Linear filters too tend to blur sharp edges, destroy lines and other fine image details, and perform poorly in the presence of signal-dependent noise. The wiener filtering [11]method requires the information about the spectra of the noise and the original signal and it works well only if the underlying signal is smooth. Wiener method implements spatial smoothing and its model complexity control correspond to choosing the window size. To over- come the weakness of the Wiener filtering, Do no ho and John stone proposed the wavelet based denoising scheme in $[12,13]$.

\section{B.Transform Domain Filtering}

The transform domain filtering methods can be subdivided according to the choice of the basic functions. The basic functions can be further classified as data adaptive and non-adaptive. Non-adaptive transforms are discussed first since they are more popular.

\section{Spatial-Frequency Filtering}

Spatial-frequency filtering refers use of low pass filters using Fast Fourier Transform (FFT). In frequency smoothing methods [11] the removal of the noise is achieved by designing a frequency domain filter 
and adapting a cut-off frequency when the noise components are deco related from the useful signal in the frequency domain. These methods are time consuming and depend on the cut-off frequency and the filter function behavior. Further more, they may produce artificial frequencies in the processed image.

\subsection{Mean filter (M.F)}

\section{Literature Survey and Related Work}

In the 1998Scott E Umbaugh, Computer Vision and Image Processing, Prentice Hall PTR, New Jersey, A mean filter acts on an image by smoothing it; that is, it reduces the intensity variation between adjacent pixels. The mean filter is nothing but a simple sliding window spatial filter that replaces the center value in the window with the average of all the neighboring pixel values including it. By doing this, it replaces pixels that are unrepresentative of their surroundings. It is implemented with a convolution mask, which provides a result that is a weighted sum of the values of a pixel and its neighbors. It is also called a linear filter. The mask or kernel is a square. Often a $3 \times 3$ square kernel is used. If the coefficients of the mask sum up to one, then the average brightness of the image is not changed. If the coefficients sum to zero, the average brightness is lost, and it returns a dark image. The mean or average filter works on the shift-multiply-sum principle [12].

\subsection{Adaptive median filter (AMF)}

In 2008, S.Saudia, Justin Varghese, Krishnan Nallaperumal, Santhosh.P.Mathew, Angelin J Robin, S.Kavitha, Proposes a new adaptive 2D spatial filter operator for the restoration of salt \& pepper impulse corrupted digital images name as -"Salt \& Pepper Impulse Detection and Median based Regularization using Adaptive Median Filter", The Adaptive Impulse Filter effectively identifies the impulsive positions with a valid impulse noise detector and replaces them by a reliable signal determined from an appropriate neighborhood. Experimental results in terms of objective metrics and visual analysis show that the proposed algorithm performs better than many of the prominent median filtering techniques reported in terms of retaining the fidelity of even highly impulse corrupted images. High objectiveness and visual reliability is provided by the new restoration algorithm at lower quantum of impulse noise also. The Adaptive Median Filter (AMF) for salt \& pepper impulse noise removal that can give much acceptable and recognizable image restoration with better visual quality at all impulse noise levels than most other median filters which develop impulse patches in the output at higher impulse noise levels. Images restored by the proposed filter for Noise ratio at $95 \%$ restoration of the Proposed Filter with better objective metrics and fidelity at higher noise ratios is an improvement in the field of impulse restoration. The computational efficiency of the proposed filter is also significant at all impulse noise ratios.

\subsection{Decision based algorithm (DBA)}

In 2009, S. Bala subramanian, S. Kalish waran, R. Muthuraj, D. Ebenezer, V. Jayaraj presented "An Efficient Non-linear Cascade Filtering Algorithm for Removal of High Density Salt and Pepper Noise in Image and Video sequence", in which an efficient non-linear cascade filter for the removal of high density salt and pepper noise in image and video is proposed. The proposed method consists of two stages to enhance the filtering. The first stage is the Decision based Median Filter (DMF), which is used to identify pixels likely to be contaminated by salt and pepper noise and replaces them by the median value. The second stage is the Unsymmetrical Trimmed Filter, either Mean Filter (UTMF) or Midpoint Filter (UTMP) which is used to trim the noisy pixels in an unsymmetrical manner and processes with the remaining pixels The basic idea is that, though the level of de-noising in the first stage is lesser at high noise densities, the second stage helps to increase the noise suppression. Hence, the proposed cascaded filter, as a whole is very suitable for low, medium as well as high noise densities even above $90 \%$. The existing non-linear filters such as Standard Median Filter (SMF), Adaptive Median Filter (AMF), Weighted Median Filter (WMF), Recursive Weighted Median Filter (RWMF) performs well only for low and medium noise densities. The recently proposed Decision Based Algorithm (DBA) shows better results up to $70 \%$ noise density and at high noise densities, the restored image quality is poor. The proposed algorithm shows better image and video quality in terms of visual appearance and quantitative measures.

\subsection{Modified decision based algorithm (MDBA)}

In 2009an improved version of DBA is used to avoid streaks in images that usually occur in DBA due to repeated replacement of the noisy pixel with neighborhood pixels. In case of MDBA noisy pixels are replaced by the median of asymmetric trimmed output. Drawback of MDBA is that under high noise densities the pixels could be all 0's or all 255's or a combination of both 0 and 255. Replacement with trimmed median value is not possible then. 
4.5Decision based unsymmetrical trimmed mean filter (DBUTMF)

In 2010 K. Aiswarya, V. Jayaraj, and D. Ebenezer, proposed a new method for removal of high density salt and pepper noise (SNP) that is - "A new and efficient algorithm for the removal of high density salt and pepper noise in images and videos," in Second Int. Conf. Computer Modeling and Simulation. To overcome the above drawback, Decision Based Algorithm (DBA) is proposed. In this, image is denoised by using a $3 \times 3$ window. If the processing pixel value is 0 or 255 it is processed or else it is left unchanged. At high noise density the median value will be 0 or 255 which is noisy. In such case, neighboring pixel is used for replacement. This repeated replacement of neighboring pixel produces streaking effect. In order to avoid this drawback, Decision Based Un symmetric Trimmed Median Filter (DBUTMF) is proposed [11],[10].

\subsection{Modified decision based unsymmetrical trimmed mean filter (MDBUTMF)}

In 2011 S. Esakkirajan, T. Veerakumar, Adabala N. Subramanyam, and C. H. Prem Chand proposed a new method for removal of high density salt and pepper noise (SNP) that is -"Removal of High Density Salt and Pepper Noise through Modified Decision Based Un-symmetric Trimmed Median Filter". Modified Decision Based Un-symmetric Trimmed Median Filter (MDBUTMF) is a non linear filter that can perform better in SAP noise removal even under high noise densities. MDBUTMF is used for the noise detection and removal process in this thesis. The filtering process consists of initially detecting noisy pixels. Each and every pixel of the image is checked for the presence of salt and pepper noise. The processing pixel is checked whether it is noisy or noise free. That is, if the processing pixel lies between maximum and minimum gray level values (between 0 and 255) then it is noise free pixel, it is left unchanged. If the processing pixel takes the maximum or minimum gray level ( 0 or 255 ) then it is noisy pixel which is processed by MDBUTMF [2].

\subsection{Modified Non-linear filter (MNLF)}

In 2013 T.Sunil kumar, A. Srinivas, M. Eswar Reddy and Dr. G. Rama chandra Reddy proposed a new method yields better results at very high noise density that is at $80 \%$ and $90 \%$ and gives better Peak Signal-toNoise Ratio (PSNR).The logic behind this paper that is Alpha Trimmed Mean Filtering(ATMF) is_symmetrical filter. As symmetric at either end even un-corrupted pixels are trimmed. This leads to loss of image details and blurring of the image. In order to overcome this drawback, an Un-symmetric Trimmed Median and Mean Filter are found. In this method, the selected 3 x 3 window elements which contain 0 's or 255's or both are removed. Then the median or mean value of remaining pixels is taken. This median or mean value is replaced in corrupted pixel value [13].

\subsection{Improved Mean Filter (IMF) -}

In 2013 Pranay Yadav, Vikas Gupta and Dilip Kumar Gandhi proposed a new algorithm has been proposed to deal with the problems, namely, poor image enhancement at high noise density, which is frequently enhanced in the IMF. In this paper improved mean filtering is used for enhancing the peak signal to noise ratio (PSNR) and image enhancement factor (IEF) both. The performances of proposed „Improved Mean

Filter "(IMF) are quantitatively vies as well as the visual and human perception vies shows better result in both conditions as compared to other existing filters .New algorithm filter also shows reliable and stable performance across a different range of noise densities varying from $10 \%-90 \%$.

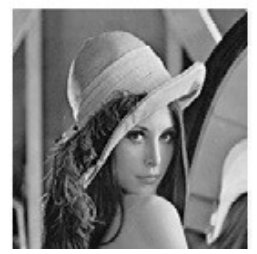

Original Image

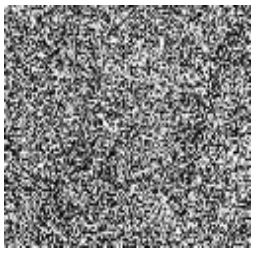

$90 \%$ Noise Density

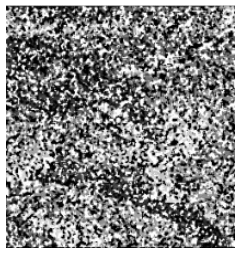

MF

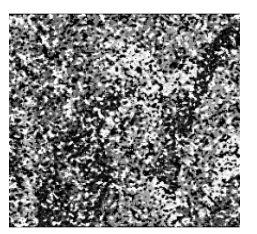

AMF

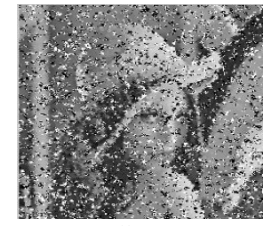

PSMF

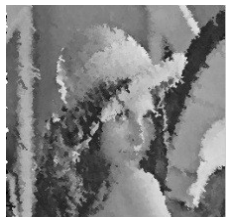

DBA 


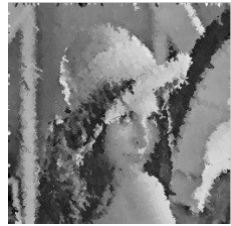

MDBA

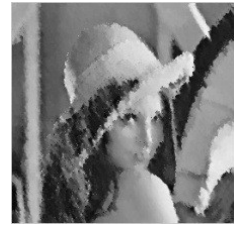

MDBUTMF

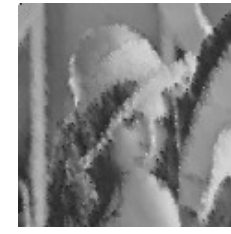

IMF

\section{Conclusion}

For simulation of proposed algorithm MATLAB 8.0 software is used. The new approach is implemented upon a 'Real Time Image' and on 'Lena' image size 256X256 as reference images for test conditions. All the test images taken are gray scale image. The test images are artificially corrupted by Salt and Pepper impulse noise of different noise density varying from $10 \%$ to $90 \%$ on MATLAB. De-noising performances are quantitatively measured by the PSNR MSE and MAE as defined in equations (3), (4) and (5) respectively:

The PSNR and MSE (Mean Square Error) can be expressed as:

psnr $=10 \log _{0} \frac{(255)^{2}}{\mathrm{mse}}$

$m s e=\frac{\sum_{i=1}^{m} \sum_{j=1}^{n}\{y(i, j)-y(i, j)\}^{2}}{m \times n}$

\section{Acknowledgment}

The authors wish to thank Mr.Virendra Patel (Director of Vedica Institute of Technology Bhopal), Prof. Pranay Yadav (He is interested in the field of image processing and wireless sensor network ). We are thankful for their valuable suggestion and timely help.

TABLE I. COMPARISON OF PSNR VALUE OF PROPOSED METHOD WITH EXISTING FILTERS FOR LENA IMAge

\begin{tabular}{|c|c|c|c|c|c|c|c|c|}
\hline $\begin{array}{c}\text { Noise } \\
\text { Density \% }\end{array}$ & MF & AMF & PSMF & DBA & MDBA & MDBUTMF & MNLF & IMF \\
\hline $\mathbf{1 0}$ & 28.4938 & 21.9845 & 30.6494 & 36.7565 & 36.7569 & 38.129 & 37.3489 & 38.70 \\
\hline $\mathbf{2 0}$ & 25.7542 & 21.9297 & 28.2089 & 33.2606 & 33.2607 & 34.6005 & 34.2358 & 35.56 \\
\hline $\mathbf{3 0}$ & 21.8465 & 21.4735 & 25.559 & 30.5659 & 30.5308 & 32.1427 & 32.1412 & 33.27 \\
\hline 40 & 18.4076 & 21.4735 & 22.6909 & 28.2609 & 28.2981 & 32.0886 & 29.0056 & 30.06 \\
\hline $\mathbf{5 0}$ & 14.734 & 20.6542 & 19.4425 & 26.2846 & 26.2503 & 28.2175 & 27.824 & 28.56 \\
\hline $\mathbf{6 0}$ & 12.2348 & 18.4092 & 12.8511 & 24.5361 & 24.6321 & 26.5937 & 26.0541 & 26.88 \\
\hline $\mathbf{7 0}$ & 9.9837 & 14.8564 & 10.5206 & 22.7798 & 22.9338 & 24.3859 & 24.3291 & 24.85 \\
\hline $\mathbf{8 0}$ & 8.0229 & 11.2968 & 8.4849 & 20.1441 & 20.4098 & 22.0101 & 22.0506 & 22.506 \\
\hline $\mathbf{9 0}$ & 6.5759 & 8.0603 & 6.7847 & 17.1205 & 17.2242 & 17.9865 & 21.325 & 21.56 \\
\hline
\end{tabular}

\section{References}

[1] R. Yang, L. Yin, M. Gabbouj, J. Astola, and Y. Neuvo, "Optimal weighted median filters understructural constraints," IEEE Trans. Signal Processing vol. 43, pp. 591-604, Mar. 1995.

[2] R. C. Hardie and K. E. Barner, "Rank conditioned rank selection filters for signal restoration," IEEE Trans. Image Processing, vol. 3, pp.192-206, Mar. 1994.

[3] A. Ben Hamza, P. Luque, J. Martinez, and R. Roman, "Removing noise and preserving details with relaxed median filters," J. Math. Imag. Vision, vol. 11, no. 2, pp. 161-177, Oct. 1999.

[4] A.K.Jain,Fundamentals of digital image processing. Prentice-Hall, 1989

[5] David L. Donoho and Iain M. Johnstone, "Ideal spatial adaption via wavelet shrinkage”, Biometrika, vol.81, pp 425-455, September 1994.

[6] David L. Donoho and Iain M. Johnstone., "Adapting to unknown smoothness via wavelet shrinkage”, Journal of the American Statistical Association, vol.90, no432, pp.1200-1224, December 1995. National Laboratory, July 27, 2001.

[7] V. Strela. "De-noising via block Wiener filtering in wavelet domain". In 3rd European Congress of Mathematics, Barcelona, July 2000. Birkhäuser Verlag.

[8] H. Choi and R. G. Baraniuk, "Analysis of wavelet domain Wiener filters," in IEEE Int. Symp. Time- Frequency and Time-Scale Analysis, (Pittsburgh), Oct. 1998. http://citeseer.ist.psu.edu/article/choi98analysis.html 
[9] H. Zhang, Aria No sratinia, and R. O. Wells, Jr., "Image de-noising via wavelet-domain spatially adaptive FIR Wiener filtering", in IEEE Proc. Int. Conf. Acoustic., Speech, Signal Processing, Istanbul, Turkey, June 2000.

[10] E. P. Simoncelli and E. H. Adelson. Noise removal via Bayesian wavelet coring. In Third Int'l Conf on Image Proc, volume I, pages 379-382, Lausanne, September 1996. IEEE Signal Proc Society.

[11] H. A. Chipman, E. D. Kolaczyk, and R. E.McCulloch: 'Adaptive Bayesian wavelet shrinkage', J.Amer. Stat. Assoc., Vol. 92, No 440, Dec. 1997, pp.1413-1421.

[12] Marteen Jansen, Ph. D. Thesis in "Wavelet thresh holding and noise reduction" 2000.

[13] M. Lang, H. Guo, J.E. Odegard, and C.S. Burrus,"Non-linear processing of a shift invariant DWT fornoise reduction," SPIE, Mathematical Imaging: Wavelet Applications for Dual Use, April 1995.

[14] I. Cohen, S. Raz and D. Malah, Translation invariant de-noising using the minimum description length criterion, Signal Processing, 75, 3, 201-223 (1999).

[15] R. G. Baraniuk, "Optimal tree approximation with wavelets," in Proc. SPIE Tech. Conf. Wavelet Applications Signal Processing VII, vol. 3813, Denver, CO, 1999, pp. 196-207. 